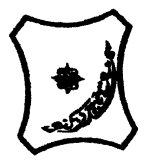

Bayero Journal of Pure and Applied Sciences, 10(2): 245 - 250

Received: November, 2017

Accepted: December, 2017

ISSN $2006-6996$

\title{
STUDIES OF Ni(II) AND Cu(II) COMPLEXES WITH SCHIFF BASE DERIVED FROM SALICYLALDEHYDE AND ANILINE
}

\author{
Hussaini, S. Y. ${ }^{1}$ Ahmad, A. ${ }^{1}$ and Sani, S. ${ }^{2}$ \\ ${ }^{1}$ Department of Chemistry, Kano University of Science and Technology, Wudil, P.M.B. 3244, Kano, \\ Nigeria. ${ }^{2}$ Department of Pure and Applied Chemistry, Usmanu Danfodiyo University, P.M.B. 2346, Sokoto, Nigeria.
}

Correspondence author: sunusiyahya69@yahoo.com

\begin{abstract}
The schiff base ligand and its complexes of divalent metal ions of $\mathrm{Ni}$ (II) and $\mathrm{Cu}$ (II) were synthesized. The ligand and the complexes were characterized by elemental analysis, potentiometry, molar conductance, melting point or decomposition temperatures and infrared analyses. The elemental analysis data show the formation of 1:2 metal-ligand ratio. The ligand and complexes were screened for antibacterial activity against Escherichia coli and Staphylococcus aureus, and antifungal activity against Aspergillums niger and Candida albican, using discs diffusion method. It has been found that the ligand and the complexes showed different activities against microorganisms. The complexes show higher activity than the free Schiff base ligand. The molar conductance of the complexes measured is low, indicating their non-electrolytic nature. The potentiometric studies of the complex compounds revealed 1:2 metal to ligand ratio.

Key words: salicylaldehyde, aniline, ligand, potentiometry, antibacterial, antifungal.
\end{abstract}

\section{INTRODUCTION}

Schiff base ligands are chemical compounds containing carbon-nitrogen double bond $(\mathrm{C}=\mathrm{N})$ functional group, called azomethine, Schiff bases are named after a German scientist, Hugo Schiff who had synthesized such compounds earlier (Hobday and Smith 1972, Holm 1966, John 2002). They are usually formed by the condensation reaction of a ketone or an aldehyde with a primary amine to generate an imine (Silver and Bassler 1967). The field of Schiff base complexes has been fast developing on account of the wide variety of possible structures for the ligands depending upon the aldehydes and amines. Schiff bases are considered as a very important class of organic compounds, which have wide application in many biological aspects. The properties of Schiff bases vary depending on the types of carbonyls and amines; they are mostly crystalline pale yellow with usually high melting points (Hassan, 1991). Schiff bases are known to form complexes with divalent or trivalent metal ions in different ratios depending on the Schiff bases that coordinate to the metal ion (Maihab et al., 2005). The chemical investigation of the Schiff base metal complexes has been carried out by used of several techniques including elemental analysis, thermogravimetric analysis, magnetic moment, infrared (IR), conductivity measurement, electronic and mass spectrometry (Hassan, 1991). Schiff base metal complexes play an essential role in the different fields, such as medicine, Agriculture, Industries, and as catalysts in a chemical reaction.

The present aim of the work is to synthesize a Schiff base derived from salicylaldehyde and aniline and to prepare its $\mathrm{Ni}(\mathrm{II})$ and $\mathrm{Cu}(\mathrm{II})$ complexes, characterize them and study their antibacterial and antifungal activities.

\section{MATERIAL AND METHODS}

All the glass wares used in this research were well washed with detergent and clean water, then rinsed with distilled water and dried in an oven at $110^{\circ} \mathrm{C}$, all the solid substances were weighed using electric mettler balance model $A B$ 54. The IR spectra were recorded in the range of $4000-400 \mathrm{~cm}^{-1}$ with an FTIR Nicolet 100 in Nujol. pH measurement was carried using Jenway $\mathrm{pH}$ meter model 3320, while molar conductance was done on the cyber can 500 model, the melting point was determined using Gallemkerm melting point apparatus. The antimicrobial activities were carried out by disc diffusion method. All the reagents used in this work were of analytical reagent (AR) grade and were used without further purification. Synthesis of Schiff Base

The Schiff base was prepared by mixing an ethanolic solution of $0.01 \mathrm{~mol}$ of salicylaldehyde $(1.22 \mathrm{~g})$ with $0.01 \mathrm{~mol}$ aniline $(0.93 \mathrm{~g})$ in the same solvent, after two hours refluxed yellow crystals appeared, the crystals were filtered and washed with ethanol and recrystallized from hot ethanol to give pure yellow crystals and dried over $\mathrm{P}_{2} \mathrm{O}_{5}$ in a desiccator (Morad et al., 2006).

Synthesis of the metal (II) Schiff Base complexes

The Schiff base complexes were synthesized by mixing $50 \mathrm{~cm}^{3}$ of ethanolic solution of the Schiff base (3.94g) with $25 \mathrm{~cm}^{3}$ of ethanolic solution of a metal (II) chloride and few drops of ammonia solution were added to adjust the $\mathrm{pH}$, the obtained mixture was refluxed for two hours and final product were obtained and recrystallized from ethanol and dried over $\mathrm{P}_{2} \mathrm{O}_{5}$ in a desiccator (Morad et. al., 2006). 
Determination of dissociation constant of Schiff base

To a $400 \mathrm{~cm}^{3}$ beaker, $90 \mathrm{~cm}^{3}$ of distilled water was added followed by $100 \mathrm{~cm}^{3}$ of $0.2 \mathrm{moldm}^{-3} \mathrm{KNO}_{3}$, $10 \mathrm{~cm}^{3}$ of a $0.4 \mathrm{moldm}^{-3}$ solution of the Schiff base and the solution of $\mathrm{NaOH}\left(0.48 \mathrm{moldm}^{-3}\right)$ was added gradually and the corresponding $\mathrm{pH}$ value recorded after each addition. The dissociation constant pka of the schiff base was calculated using the equation below. (Gregory et al., 1978)

where $\mathrm{p}^{\mathrm{K}} \mathrm{a}$

$$
\mathrm{p}^{\mathrm{K}} \mathrm{a}=-\log \left[\mathrm{H}^{+}\right]+\log \left[A_{t o t}-\frac{\left[N_{a}^{+}\right]+\left[\mathrm{H}^{+}\right]-\left[\mathrm{OH}^{-}\right]}{\left[N_{a}^{+}\right]+\left[\mathrm{H}^{+}\right]-\left[\mathrm{OH}^{-}\right]}\right]
$$

$$
\begin{array}{ll}
=\text { Dissociation constant } \\
{\left[\mathrm{H}^{+}\right] \quad=\text { Hydrogen ion concentration }} \\
{\left[\mathrm{OH}^{-}\right] \quad=\text { Hydroxyl ion concentration }} \\
{\left[\mathrm{Na}^{+}\right] \quad=\text { Sodium ion concentration }} \\
\mathrm{A}_{\text {tot }} \quad=\text { A total }
\end{array}
$$

Determination of dissociation constant of the complexes

Into a $400 \mathrm{~cm}^{3}$ beaker $100 \mathrm{~cm}^{3}$ of $0.2 \mathrm{M} \mathrm{KNO}_{3}$, $1 \mathrm{mmole}$ of $\mathrm{Ni}(\mathrm{II})$ or $\mathrm{Cu}(\mathrm{II})$ chloride, $0.1 \mathrm{M} \mathrm{HNO}_{3}$ and $90 \mathrm{~cm}^{3}$ of distilled water were added, respectively. A magnetic stirring bar and the sodium salt of the Schiff base prepared by neutralizing a known quantity of the
Schiff base with the calculated amount of standardized $\mathrm{NaOH}$ solution. After each $0.2 \mathrm{~cm}^{3}$ aliquot addition, the corresponding $\mathrm{pH}$ of the stirred reaction mixture was recorded. From the result obtained, the number of coordinated ligands per metal ion of each metal (II) schiff base complex was calculated using the expression below (Avar.et. al., 1975).

Where $n=$ Number of coordinated ligands

$$
\boldsymbol{n}=\frac{A_{\text {tot }}-\left[1+\frac{\mathrm{Ka}}{\mathrm{H}^{+}}\right]\left[(\mathrm{CH})+\left[\mathrm{OH}^{-}\right]-\left[\mathrm{OH}^{+}\right]\right.}{M_{\text {tot }}}
$$

$$
\begin{aligned}
& \mathrm{A}_{\text {tot }}=\mathrm{A} \text { total } \\
& {\left[\mathrm{H}^{+}\right] \quad=\text { Hydrogen ion concentration }} \\
& {\left[\mathrm{OH}^{-}\right] \quad=\text { Hydroxyl ion concentration }} \\
& \mathrm{M}_{\text {tot }}=\mathrm{M} \text { total }
\end{aligned}
$$

\section{Antibacterial and Antifungal activity}

The paper discs were impregnated with 1000, 2000 and $3000 \mu \mathrm{g} / \mathrm{ml}$ of the Schiff base and its metal (II) complexes. Two loops of the standard inoculums were evenly streaked on the plates in duplicates. Discs containing the impregnated quantities of the complexes as well as the control discs (with only DMSO) were placed firmly on the surface of the medium by means of sterile syringe needle at $40 \mathrm{~mm}$

\begin{tabular}{|c|c|c|c|c|c|}
\hline Compound & Molecular weight & Colour & $\begin{array}{c}\text { Melting } \\
\text { point }\left({ }^{\circ} \mathrm{C}\right)\end{array}$ & $\begin{array}{l}\text { Decompositio } \\
\text { n Temp. }\left({ }^{0} \mathrm{C}\right)\end{array}$ & $\begin{array}{l}\text { Yield } \\
(\%)\end{array}$ \\
\hline Ligand $\left(\mathrm{C}_{13} \mathrm{H}_{10} \mathrm{NO}\right)$ & 197.00 & Yellow & 104 & - & 94.92 \\
\hline$\left[\mathrm{NiL}_{2}\right] .2 \mathrm{H}_{2} \mathrm{O}$ & 629.71 & Yellow & - & $132^{\circ} \mathrm{C}$ & 54.88 \\
\hline$\left[\mathrm{CuL}_{2}\right] \cdot 2 \mathrm{H}_{2} \mathrm{O}$ & 562.48 & Black & - & $158^{\circ} \mathrm{C}$ & 55.30 \\
\hline
\end{tabular}

apart. For the bacteria, the plates were incubated at $37^{\circ} \mathrm{C}$ for one day (shamsuddeen et al., 2008). For the fungal activity, it was incubated at room temperature for two days (Hassan, et al., 2006). Each of the plates was examined for the clear zone of inhibitions. The diameters of the zone of inhibitions were measured with millimeter rule and the mean recorded in the nearest millimeter.

RESULTS AND DISCUSSION

Table 1: Molecular weight, colour and percentage yield of the ligand and metal complexes

Where $L=$ schiff base ligand

Table 2: percentage compositions of metal ion, ligand, and water of crystallization.

\begin{tabular}{cccc}
\hline Complexes & Metal (\%) & Ligand (\%) & Water of crystallization (\%) \\
\hline$\left[\mathrm{Ni}(\mathrm{L})_{2}\right] 2 \mathrm{H}_{2} \mathrm{O}$ & 11.04 & 81.26 & 7.70 \\
{$\left[\mathrm{Cu}(\mathrm{L})_{2}\right] 2 \mathrm{H}_{2} \mathrm{O}$} & 12.10 & 78.05 & 9.85 \\
\hline
\end{tabular}

Table 3: Solubility of the ligand and its complexes in some common solvents

\begin{tabular}{ccccccccccc}
\hline Compound & DMSO & Meth & Water & Toluene & Ethanol & Acetone & Ether & Ben & Isopropanol & DE \\
\hline Ligand $\left(\mathrm{C}_{13} \mathrm{H}_{10} \mathrm{NO}\right)$ & $\mathrm{S}$ & $\mathrm{S}$ & IS & SS & $\mathrm{S}$ & IS & IS & IS & IS & IS \\
{$\left[\mathrm{NiL}_{2}\right] .2 \mathrm{H}_{2} \mathrm{O}$} & $\mathrm{S}$ & $\mathrm{S}$ & IS & SS & IS & IS & IS & IS & IS & IS \\
{$\left[\mathrm{CuL}_{2}\right] .2 \mathrm{H}_{2} \mathrm{O}$} & $\mathrm{S}$ & $\mathrm{S}$ & IS & SS & $\mathrm{S}$ & $\mathrm{S}$ & IS & IS & IS & IS
\end{tabular}

Key: S = Soluble. SS = slightly soluble. IS = Insoluble, DE = diethyl ether, Meth = Methanol, Ben = Benzene 
Bajopas Volume 10 Number 2 December, 2017

Table 4: Conductivity measurement of the complex compounds

\begin{tabular}{|c|c|c|c|}
\hline Complexes & $\begin{array}{c}\text { Concentration } \\
\text { moldm }^{-3}\end{array}$ & $\begin{array}{l}\text { Electrical conductivity } \mathrm{ohm}^{-1} \\
\mathrm{~cm}^{-1}\end{array}$ & $\begin{array}{c}\text { Molar conductivity ohm }{ }^{-1} \mathrm{~cm}^{2} \\
\mathrm{~mol}^{-1}\end{array}$ \\
\hline$\left[\mathrm{NiL}_{2}\right] \cdot 2 \mathrm{H}_{2} \mathrm{O}$ & $1 \times 10^{-3}$ & $9.40 \times 10^{-6}$ & 94.0 \\
\hline$\left[\mathrm{CuL}_{2}\right] \cdot 2 \mathrm{H}_{2} \mathrm{O}$ & $1 \times 10^{-3}$ & $6.85 \times 10^{-6}$ & 68.5 \\
\hline
\end{tabular}

Table 5: Infrared spectral data of the Schiff base and its metal complexes

\begin{tabular}{|c|c|c|c|c|c|}
\hline Compound & $V(C=N) c^{-1}$ & $v(C-0) \mathrm{cm}^{-1}$ & $V(M-N) c^{-1}$ & $V(M-0) \mathrm{cm}^{-1}$ & $V(O-H) \mathrm{cm}^{-1}$ \\
\hline Ligand $\left(\mathrm{C}_{13} \mathrm{H}_{10} \mathrm{NO}\right)$ & 1620 & 1210 & - & - & 3460 \\
\hline$\left[\mathrm{NiL}_{2}\right] \cdot 2 \mathrm{H}_{2} \mathrm{O}$ & 1580 & 1240 & 680 & 550 & - \\
\hline$\left[\mathrm{CuL}_{2}\right] \cdot 2 \mathrm{H}_{2} \mathrm{O}$ & 1590 & 1270 & 560 & 440 & - \\
\hline
\end{tabular}

Table 6: The stability constant and Gibbs free energy $(\Delta G)$ of the metal complexes Complex Stability constants Gibb's Free energy DG $\left(\mathrm{KJmol}^{-1}\right)$

$\begin{array}{lll}{\left[\mathrm{NiL}_{2}\right] .2 \mathrm{H}_{2} \mathrm{O}} & 5.01 \times 10^{18} & -106.6 \\ {\left[\mathrm{CuL}_{2}\right] .2 \mathrm{H}_{2} \mathrm{O}} & 8.32 \times 10^{21} & -125.0\end{array}$

Table 7: Antibacterial effect of Schiff base and complexes

Compound Escherichia coli Staphylococcus aureus

\begin{tabular}{ccccccc} 
& $1000 \mathrm{ug} / \mathrm{ml}$ & $2000 \mathrm{ug} / \mathrm{ml}$ & $3000 \mathrm{ug} / \mathrm{ml}$ & $1000 \mathrm{ug} / \mathrm{ml}$ & $2000 \mathrm{ug} / \mathrm{ml}$ & $3000 \mathrm{ug} / \mathrm{ml}$ \\
\hline Ligand $\left(\mathrm{C}_{13} \mathrm{H}_{10} \mathrm{NO}\right)$ & - & - & - & - & - & - \\
{$\left[\mathrm{NiL}_{2}\right] .2 \mathrm{H}_{2} \mathrm{O}$} & - & - & + & - & - & + \\
{$\left[\mathrm{CuL}_{2}\right] .2 \mathrm{H}_{2} \mathrm{O}$} & + & + & ++ & - & + & + \\
\hline
\end{tabular}

Table 8: Antifungal effect of Schiff base and its metal complexes

\section{Compound Aspergillus niger Candida albican}

\begin{tabular}{ccccccc} 
& $1000 \mathrm{ug} / \mathrm{ml}$ & $2000 \mathrm{ug} / \mathrm{ml}$ & $3000 \mathrm{ug} / \mathrm{ml}$ & $1000 \mathrm{ug} / \mathrm{ml}$ & $2000 \mathrm{ug} / \mathrm{ml}$ & $3000 \mathrm{ug} / \mathrm{ml}$ \\
\hline Ligand & - & + & ++ & - & + & +++ \\
$\left(\mathrm{C}_{13} \mathrm{H}_{10} \mathrm{NO}\right)$ & & & & & & - \\
{$\left[\mathrm{NiL}_{2}\right] .2 \mathrm{H}_{2} \mathrm{O}$} & + & ++ & +++ & - & + \\
{$\left[\mathrm{CuL}_{2}\right] .2 \mathrm{H}_{2} \mathrm{O}$} & - & - & + & + & ++ & ++ \\
\hline
\end{tabular}

Key: $\quad$ High active $=+++($ Inhibition zone $>12 \mathrm{~mm})$ Moderate active $=++($ Inhibition zone $9-12 \mathrm{~mm})$ Slightly active $=+($ Inhibition zone7 $-9 \mathrm{~mm})$

Inactive $=-($ Inhibition zone $\leq 6 \mathrm{~mm})$

Table 9: Dissociation constant (pKa) of the Schiff base

\begin{tabular}{|c|c|c|c|c|c|c|c|}
\hline $\mathbf{S} / \mathbf{N}$ & $\begin{array}{c}\text { Vol. of } \\
\text { NaOH } \\
\left(\mathrm{cm}^{3}\right)\end{array}$ & pH & {$\left[\mathbf{H}^{+}\right]$} & {$\left[\mathrm{OH}^{-}\right]$} & {$\left[\mathrm{Na}^{+}\right]$} & $A_{\text {tot }}$ & Pka \\
\hline 1 & 0.5 & 9.30 & $3.3535 \times 10^{-10}$ & $2.1577 \times 10^{-5}$ & $1.2070 \times 10^{-3}$ & $1.9950 \times 10^{-2}$ & 10.6730 \\
\hline 2 & 1.0 & 10.33 & $3.1297 \times 10^{-11}$ & $2.3121 \times 10^{-4}$ & $2.4080 \times 10^{-3}$ & $1.9900 \times 10^{-2}$ & 11.4152 \\
\hline 3 & 1.5 & 10.95 & $7.5076 \times 10^{-12}$ & $9.6382 \times 10^{-4}$ & $3.6030 \times 10^{-3}$ & $1.9851 \times 10^{-2}$ & 11.9389 \\
\hline 4 & 2.0 & 11.21 & $4.1257 \times 10^{-12}$ & $1.7539 \times 10^{-3}$ & $4.7921 \times 10^{-3}$ & $1.9802 \times 10^{-2}$ & 12.1263 \\
\hline 5 & 2.5 & 11.37 & $2.8543 \times 10^{-12}$ & $2.5351 \times 10^{-3}$ & $5.9753 \times 10^{-3}$ & $1.9753 \times 10^{-2}$ & 12.2205 \\
\hline 6 & 3.0 & 11.47 & $2.2673 \times 10^{-12}$ & $3.1915 \times 10^{-3}$ & $7.1577 \times 10^{-3}$ & $1.9764 \times 10^{-2}$ & 12.2438 \\
\hline 7 & 3.5 & 11.54 & $1.9297 \times 10^{-12}$ & $3.7497 \times 10^{-3}$ & $8.3243 \times 10^{-3}$ & $1.9656 \times 10-2$ & 12.2326 \\
\hline 8 & 4.0 & 11.61 & $1.6425 \times 10^{-12}$ & $4.4055 \times 10^{-3}$ & $9.4902 \times 10^{-3}$ & $1.9608 \times 10^{-2}$ & 12.2403 \\
\hline 9 & 4.5 & 11.68 & $1.3980 \times 10^{-2}$ & $5.1761 \times 10^{-3}$ & $1.0650 \times 10^{-2}$ & $1.9560 \times 10^{-2}$ & 12.2650 \\
\hline 10 & 5.0 & 11.72 & $1.2750 \times 10^{-12}$ & $5.6754 \times 10^{-3}$ & $1.1805 \times 10^{-2}$ & $1.9512 \times 10^{-2}$ & 12.2336 \\
\hline 11 & 5.5 & 11.75 & $1.1899 \times 10^{-12}$ & $6.0814 \times 10^{-3}$ & $1.2954 \times 10^{-2}$ & $1.9465 \times 10^{-2}$ & 12.1875 \\
\hline 12 & 6.0 & 11.80 & $1.0605 \times 10^{-12}$ & $6.8234 \times 10^{-3}$ & $1.4097 \times 10^{-2}$ & $1.9417 \times 10^{-12}$ & 12.1971 \\
\hline 13 & 6.5 & 11.84 & $9.6716 \times 10^{-13}$ & $7.4817 \times 10-3$ & $1.5235 \times 10^{-2}$ & $1.9370 \times 10^{-2}$ & 12.1727 \\
\hline 14 & 7.0 & 11.87 & $9.0261 \times 10^{-13}$ & $8.0168 \times 10^{-3}$ & $1.6367 \times 10^{-2}$ & $1.9324 \times 10^{-2}$ & 12.1632 \\
\hline 15 & 7.5 & 11.90 & $8.4236 \times 10^{-13}$ & $8.5901 \times 10^{-3}$ & $1.7493 \times 10^{-2}$ & $1.9277 \times 10^{-2}$ & 12.1409 \\
\hline 16 & 8.0 & 11.92 & $8.0445 \times 10^{-13}$ & $8.9950 \times 10^{-3}$ & $1.8615 \times 10^{-2}$ & $1.9231 \times 10^{-2}$ & 12.0941 \\
\hline 17 & 8.5 & 11.93 & $7.8614 \times 10^{-13}$ & $9.2045 \times 10^{-3}$ & $1.9731 \times 10^{-2}$ & $1.9185 \times 10^{-2}$ & 12.0196 \\
\hline 18 & 9.0 & 11.95 & $7.5076 \times 10^{-13}$ & $9.6383 \times 10^{-3}$ & $2.0842 \times 10^{-2}$ & $1.9139 \times 10^{-2}$ & 11.9747 \\
\hline 19 & 9.5 & 11.97 & $7.1697 \times 10^{-13}$ & $1.0093 \times 10^{-2}$ & $2.1947 \times 10^{-2}$ & $1.9093 \times 10^{-2}$ & 11.9303 \\
\hline 20 & 10 & 11.99 & $6.8470 \times 10^{-13}$ & $1.0568 \times 10^{-2}$ & $2.3048 \times 10^{-2}$ & $1.9048 \times 10^{-2}$ & 11.8857 \\
\hline
\end{tabular}

Average pka Value $=12.0178$ 
Bajopas Volume 10 Number 2 December, 2017

Table 10: Estimation of number of coordinated Schiff base per Nickel (II) complex

\begin{tabular}{|c|c|c|c|c|c|c|c|}
\hline $\mathbf{S} / \mathbf{N}$ & $\begin{array}{l}\text { Volume of sodium } \\
\text { liganate }\left(\mathrm{cm}^{3}\right)\end{array}$ & $\mathbf{P}^{\mathbf{H}}$ & {$\left[\mathrm{H}^{+}\right]$} & {$\left[\mathrm{OH}^{-}\right]$} & $\log [A]$ & $M_{\text {total }}$ & $\mathbf{N}$ \\
\hline 1 & 2.2 & 4.76 & $1.16 \times 10^{-5}$ & $6.22 \times 10^{-10}$ & -10.09 & $4.95 \times 10^{-3}$ & 0.6801 \\
\hline 2 & 2.4 & 4.86 & $9.24 \times 10^{-6}$ & $7.83 \times 10^{-10}$ & -9.99 & $4.94 \times 10^{-3}$ & 0.7595 \\
\hline 3 & 2.6 & 5.06 & $5.83 \times 10^{-6}$ & $1.24 \times 10^{-9}$ & -9.79 & $4.94 \times 10^{-3}$ & 0.8385 \\
\hline 4 & 2.8 & 5.27 & $3.59 \times 10^{-6}$ & $2.01 \times 10^{-9}$ & -9.57 & $4.93 \times 10^{-3}$ & 0.9179 \\
\hline 5 & 3.0 & 5.49 & $2.17 \times 10^{-6}$ & $3.34 \times 10^{-9}$ & -9.36 & $4.93 \times 10^{-3}$ & 0.9973 \\
\hline 6 & 3.2 & 5.75 & $1.19 \times 10^{-6}$ & $6.08 \times 10^{-9}$ & -8.92 & $4.92 \times 10^{-3}$ & 1.0769 \\
\hline 7 & 3.4 & 5.93 & $7.86 \times 10^{-7}$ & $9.20 \times 10^{-9}$ & -8.91 & $4.92 \times 10^{-3}$ & 1.1567 \\
\hline 8 & 3.6 & 6.11 & $5.19 \times 10^{-7}$ & $1.39 \times 10^{-8}$ & -8.73 & $4.91 \times 10^{-3}$ & 1.2366 \\
\hline 9 & 3.8 & 6.24 & $3.85 \times 10^{-7}$ & $1.88 \times 10^{-8}$ & -8.60 & $4.91 \times 10^{-3}$ & 1.3162 \\
\hline 10 & 4.0 & 6.37 & $2.85 \times 10^{-7}$ & $2.53 \times 10^{-8}$ & -8.47 & $4.90 \times 10^{-3}$ & 1.3960 \\
\hline 11 & 4.2 & 6.48 & $2.22 \times 10^{-7}$ & $3.27 \times 10^{-8}$ & -8.36 & $4.90 \times 10^{-3}$ & 1.4758 \\
\hline 12 & 4.4 & 6.57 & $1.80 \times 10^{-7}$ & $4.02 \times 10^{-8}$ & -8.27 & $4.89 \times 10^{-3}$ & 1.5557 \\
\hline 13 & 4.6 & 6.63 & $1.57 \times 10^{-7}$ & $4.61 \times 10^{-8}$ & -8.21 & $4.89 \times 10^{-3}$ & 1.6362 \\
\hline 14 & 4.8 & 6.69 & $1.37 \times 10^{-7}$ & $5.30 \times 10^{-8}$ & -8.15 & $4.88 \times 10^{-3}$ & 1.7152 \\
\hline 15 & 5.0 & 6.73 & $1.25 \times 10^{-7}$ & $5.81 \times 10^{-8}$ & -8.11 & $4.88 \times 10^{-3}$ & 1.7950 \\
\hline 16 & 5.2 & 6.79 & $1.09 \times 10^{-7}$ & $6.67 \times 10^{-8}$ & -8.06 & $4.87 \times 10^{-3}$ & 1.8747 \\
\hline 17 & 5.4 & 6.85 & $9.45 \times 10^{-8}$ & $7.66 \times 10^{-8}$ & -7.99 & $4.87 \times 10^{-3}$ & 1.9546 \\
\hline 18 & 5.6 & 6.90 & $8.42 \times 10^{-8}$ & $8.59 \times 10^{-8}$ & -7.95 & $4.86 \times 10^{-3}$ & 2.0344 \\
\hline 19 & 5.8 & 6.94 & $7.68 \times 10^{-8}$ & $9.42 \times 10^{-8}$ & -7.90 & $4.86 \times 10^{-3}$ & 2.1142 \\
\hline 20 & 6.0 & 6.98 & $7.01 \times 10^{-8}$ & $1.03 \times 10^{-7}$ & -7.86 & $4.85 \times 10^{-3}$ & 2.1918 \\
\hline 21 & 6.2 & 7.04 & $6.10 \times 10^{-8}$ & $1.19 \times 10^{-7}$ & -7.80 & $4.85 \times 10^{-3}$ & 2.2737 \\
\hline 22 & 6.4 & 7.11 & $5.19 \times 10^{-8}$ & $1.39 \times 10^{-7}$ & -7.73 & $4.85 \times 10^{-3}$ & 2.3536 \\
\hline 23 & 6.6 & 7.14 & $4.85 \times 10^{-8}$ & $1.49 \times 10^{-7}$ & -7.68 & $4.84 \times 10^{-3}$ & 2.4333 \\
\hline 24 & 6.8 & 7.17 & $4.52 \times 10^{-8}$ & $1.60 \times 10^{-7}$ & -7.67 & $4.84 \times 10^{-3}$ & 2.5152 \\
\hline 25 & 7.0 & 7.22 & $4.03 \times 10^{-8}$ & $1.79 \times 10^{-7}$ & -7.62 & $4.83 \times 10^{-3}$ & 2.5931 \\
\hline 26 & 7.2 & 7.26 & $3.68 \times 10^{-8}$ & $1.97 \times 10^{-7}$ & -7.58 & $4.83 \times 10^{-3}$ & 2.6726 \\
\hline 27 & 7.4 & 7.32 & $3.20 \times 10^{-8}$ & $2.26 \times 10^{-7}$ & -7.52 & $4.82 \times 10^{-3}$ & 2.7526 \\
\hline 28 & 7.6 & 7.37 & $2.85 \times 10^{-8}$ & $2.54 \times 10^{-7}$ & -7.47 & $4.82 \times 10^{-3}$ & 2.8324 \\
\hline 29 & 7.8 & 7.47 & $2.54 \times 10^{-8}$ & $2.84 \times 10^{-7}$ & -7.42 & $4.81 \times 10^{-3}$ & 2.9121 \\
\hline 30 & 8.0 & 7.52 & $2.32 \times 10^{-8}$ & $3.12 \times 10^{-7}$ & -7.38 & $4.81 \times 10^{-3}$ & 2.9495 \\
\hline
\end{tabular}

Table 11: Estimation of number of coordinated Schiff base per Copper (II) complex

\begin{tabular}{|c|c|c|c|c|c|c|c|}
\hline $\mathbf{S} / \mathbf{N}$ & $\begin{array}{l}\text { Volume of sodium } \\
\text { liganate }\left(\mathrm{cm}^{3}\right)\end{array}$ & $\mathbf{p}^{H}$ & {$\left[\mathrm{H}^{+}\right]$} & {$\left[\mathrm{OH}^{-}\right]$} & $\log [A]$ & $M_{\text {total }}$ & $\mathbf{N}$ \\
\hline 1 & 2.2 & 3.57 & $1.80 \times 10^{-4}$ & $4.02 \times 10^{-11}$ & -11.35 & $4.95 \times 10^{-3}$ & 0.7142 \\
\hline $\begin{array}{l}2 \\
3\end{array}$ & $\begin{array}{l}2.4 \\
2.6\end{array}$ & $\begin{array}{l}3.72 \\
3.83\end{array}$ & $\begin{array}{l}1.28 \times 10^{-4} \\
9.90 \times 10^{-5}\end{array}$ & $\begin{array}{l}5.68 \times 10^{-11} \\
7.31 \times 10^{-11}\end{array}$ & $\begin{array}{l}-11.18 \\
-11.06\end{array}$ & $\begin{array}{l}4.94 \times 10^{-3} \\
4.94 \times 10^{-3}\end{array}$ & $\begin{array}{l}0.7834 \\
0.8587\end{array}$ \\
\hline 4 & 2.8 & 3.88 & $8.82 \times 10^{-5}$ & $8.20 \times 10^{-11}$ & -11.00 & $4.93 \times 10^{-3}$ & 0.9351 \\
\hline 5 & 3.0 & 3.94 & $7.68 \times 10^{-5}$ & $9.42 \times 10^{-11}$ & -10.94 & $4.93 \times 10^{-3}$ & 1.0125 \\
\hline 6 & 3.2 & 3.99 & $6.85 \times 10^{-5}$ & $1.06 \times 10^{-10}$ & -10.88 & $4.92 \times 10^{-3}$ & 1.0907 \\
\hline $\begin{array}{l}7 \\
8\end{array}$ & $\begin{array}{l}3.4 \\
3.6\end{array}$ & $\begin{array}{l}4.03 \\
4.06\end{array}$ & $\begin{array}{l}6.24 \times 10^{-5} \\
5.83 \times 10^{-5}\end{array}$ & $\begin{array}{l}1.16 \times 10^{-10} \\
1.24 \times 10^{-10}\end{array}$ & $\begin{array}{l}-10.84 \\
-10.81\end{array}$ & $\begin{array}{l}4.92 \times 10^{-3} \\
4.91 \times 10^{-3}\end{array}$ & $\begin{array}{l}1.1692 \\
1.2483\end{array}$ \\
\hline 9 & 3.8 & 4.09 & $5.44 \times 10^{-5}$ & $1.33 \times 10^{-10}$ & -10.78 & $4.91 \times 10^{-3}$ & 1.3272 \\
\hline $\begin{array}{l}10 \\
11\end{array}$ & $\begin{array}{l}4.0 \\
4.2\end{array}$ & $\begin{array}{l}4.12 \\
4.13\end{array}$ & $\begin{array}{l}5.08 \times 10^{-5} \\
4.96 \times 10^{-5}\end{array}$ & $\begin{array}{l}1.43 \times 10^{-10} \\
1.46 \times 10^{-10}\end{array}$ & $\begin{array}{l}-10.75 \\
-10.74\end{array}$ & $\begin{array}{l}4.90 \times 10^{-3} \\
4.90 \times 10^{-3}\end{array}$ & $\begin{array}{l}1.4063 \\
1.4859\end{array}$ \\
\hline 12 & 4.4 & 4.14 & $4.85 \times 10^{-5}$ & $1.49 \times 10^{-10}$ & -10.73 & $4.89 \times 10^{-3}$ & 1.5656 \\
\hline 13 & 4.6 & 4.17 & $4.52 \times 10^{-5}$ & $1.60 \times 10^{-10}$ & -10.69 & $4.89 \times 10^{-3}$ & 1.6446 \\
\hline 14 & 4.8 & 4.19 & $4.32 \times 10^{-5}$ & $1.67 \times 10^{-10}$ & -10.67 & $4.88 \times 10^{-3}$ & 1.7141 \\
\hline 15 & 5.0 & 4.20 & $4.22 \times 10^{-5}$ & $1.71 \times 10^{-10}$ & -10.66 & $4.88 \times 10^{-3}$ & 1.8037 \\
\hline $\begin{array}{l}16 \\
17\end{array}$ & $\begin{array}{l}5.2 \\
5.4\end{array}$ & $\begin{array}{l}4.24 \\
4.26\end{array}$ & $\begin{array}{l}3.85 \times 10^{-5} \\
3.68 \times 10^{-5}\end{array}$ & $\begin{array}{l}1.88 \times 10^{-10} \\
1.97 \times 10^{-10}\end{array}$ & $\begin{array}{l}-10.62 \\
-10.60\end{array}$ & $\begin{array}{l}4.87 \times 10^{-3} \\
4.87 \times 10^{-3}\end{array}$ & $\begin{array}{l}1.8826 \\
1.9621\end{array}$ \\
\hline 18 & 5.6 & 4.28 & $3.51 \times 10^{-5}$ & $2.06 \times 10^{-10}$ & -10.58 & $4.86 \times 10^{-3}$ & 2.0416 \\
\hline 19 & 5.8 & 4.30 & $3.35 \times 10^{-5}$ & $2.16 \times 10^{-10}$ & -10.56 & $4.86 \times 10^{-3}$ & 2.1211 \\
\hline 20 & 6.0 & 4.34 & $3.06 \times 10^{-5}$ & $2.37 \times 10^{-10}$ & -10.52 & $4.85 \times 10^{-3}$ & 2.2002 \\
\hline 21 & 6.2 & 4.35 & $2.99 \times 10^{-5}$ & $2.42 \times 10^{-10}$ & -10.51 & $4.85 \times 10^{-3}$ & 2.2799 \\
\hline 22 & 6.4 & 4.36 & $2.92 \times 10^{-5}$ & $2.48 \times 10^{-10}$ & -10.50 & $4.85 \times 10^{-3}$ & 2.3596 \\
\hline 23 & 6.6 & 4.37 & $2.85 \times 10^{-5}$ & $2.54 \times 10^{-10}$ & -10.49 & $4.84 \times 10^{-3}$ & 2.4392 \\
\hline 24 & 6.8 & 4.39 & $2.73 \times 10^{-5}$ & $2.65 \times 10^{-10}$ & -10.47 & $4.84 \times 10^{-3}$ & 2.5189 \\
\hline 25 & 7.0 & 4.41 & $2.60 \times 10^{-5}$ & $2.78 \times 10^{-10}$ & -10.44 & $4.83 \times 10^{-3}$ & 2.5985 \\
\hline $\begin{array}{l}26 \\
27\end{array}$ & $\begin{array}{l}7.2 \\
7.4\end{array}$ & $\begin{array}{l}4.42 \\
4.43\end{array}$ & $\begin{array}{l}2.54 \times 10^{-5} \\
2.48 \times 10^{-5}\end{array}$ & $\begin{array}{l}2.84 \times 10^{-10} \\
2.91 \times 10^{-10}\end{array}$ & $\begin{array}{l}-10.43 \\
-10.42\end{array}$ & $\begin{array}{l}4.83 \times 10^{-3} \\
4.82 \times 10^{-3}\end{array}$ & $\begin{array}{l}2.6779 \\
2.7576\end{array}$ \\
\hline $\begin{array}{l}28 \\
29 \\
30\end{array}$ & $\begin{array}{l}7.6 \\
7.8 \\
8.0\end{array}$ & $\begin{array}{l}4.46 \\
4.47 \\
4.49\end{array}$ & $\begin{array}{l}2.32 \times 10^{-5} \\
2.27 \times 10^{-5} \\
2.17 \times 10^{-5}\end{array}$ & $\begin{array}{l}3.12 \times 10^{-10} \\
3.19 \times 10^{-10} \\
3.34 \times 10^{-10}\end{array}$ & $\begin{array}{l}-10.39 \\
-10.38 \\
-10.36\end{array}$ & $\begin{array}{l}4.82 \times 10^{-3} \\
4.81 \times 10^{-3} \\
4.81 \times 10^{-3}\end{array}$ & $\begin{array}{l}2.8373 \\
2.9168 \\
2.9966\end{array}$ \\
\hline
\end{tabular}


The interaction between Salicylaldehyde and aniline gave a yellowcrystalline schiff base. The percentage yield and melting point of the schiff base are $94.92 \%$ and $104^{\circ} \mathrm{C}$, respectively. The reaction of schiff base with $\mathrm{Ni}$ (II) and $\mathrm{Cu}$ (II) chlorides gave metal (II) complexes with the following percentage yields $54.88 \%$ and $55.30 \%$, respectively. $\mathrm{Cu}$ (II) and $\mathrm{Ni}$ (II) schiff base complexes are yellow and black crystals with the decomposition temperature of $132^{\circ} \mathrm{C}$ and $158^{\circ} \mathrm{C}$, respectively (Table 1 ). The colour of complexes is due to the electronic excitation from low $t_{2 g}$ to higher $e_{g}$ by absorption of visible light. (Nazeeruddin and Gratzel, 2007)

The percentage composition of the metal ion, ligand and water content of each metal (II) schiff base complex determined, revealed the general formula $\left[\mathrm{ML}_{2}\right] . \mathrm{nH}_{2} \mathrm{O}$. (Table 2). The solubility test of the schiff base and its complex compounds (Table 3 ) generally showed good solubility in DMSO and methanol. Cu (II) complex is soluble in ethanol and acetone. However, the schiff base ligand and its complexes recorded poor solubility in water and some organic solvent such as Toluene, ether, benzene, diethyl ether, and isopropanol. The molar conductance of synthesized complexes (Table 4) were measured using $10^{-3} \mathrm{M}$ methanol and DMSO and the values are 94.0 and $68.5 \mathrm{Ohm}^{-1} \mathrm{~cm}^{2} \mathrm{~mol}^{-1}$ respectively. These values are too low to account for any dissociation of the complexes and the obtained values were taken as a good evidence of the existence of a non-electrolytic nature of the complexes (Geary, 1972).

The FT-IR frequencies exhibited by the schiff base ligand and their complexes are tabulated in Table 5. The free schiff base absorbs in the region $3460 \mathrm{~cm}^{-1}$ which is assigned to $(\mathrm{O}-\mathrm{H})$ stretching vibration which did not appeared in the complexes, indicating the coordination through the metal atom. The bands at $1620 \mathrm{~cm}^{-1}$ shown by ligand are attributed to $\mathrm{V}(\mathrm{C}=\mathrm{N})$ which has been shifted toward lower region at 1580 and $1590 \mathrm{~cm}^{-1}$ in the respective complexes, indicating the participation of the azomethine group in the complexation, this corroborate with what Makodeand Aswa,(2004) reported. The band at $1210 \mathrm{~cm}^{-1}$ are due to $(\mathrm{C}-\mathrm{O})$ stretching frequencies, however, a Shift to higher frequency was observed in the complexes. $\mathrm{Ni}(\mathrm{II})$ and $\mathrm{Cu}$ (II) complexes showed bands at lower frequency region of $560 \mathrm{~cm}^{-1}$ and $680 \mathrm{~cm}^{-1}$ assigned to (M-N) respectively while440 $\mathrm{cm}^{-1}$ and $550 \mathrm{~cm}^{-1}$ are also assigned to respective $(\mathrm{M}-\mathrm{O})$ stretching vibration (Saleen et al., 2003), confirming coordination of the ligands to the respective metals. (Table 5).

The dissociation constant (pKa) of the schiff base is 12.0178 suggesting weak base (Table 9 ). The stability constants of $\mathrm{Ni}$ (II) and $\mathrm{Cu}$ (II) schiff base complexes determined are $5.01 \times 10^{18}$ and $8.32 \times 10^{21}$ respectively, indicating good stability of the complexes, supported by high decomposition temperature shown in Table 1. The Gibbs free energy $(\Delta G)$ of the schiff base complexes fall in the range of -106.6 and -125.0 $\mathrm{KJmol}^{-1}$ are relatively low suggesting good stability for the complex compounds. The magnitude of $\Delta G$ indicates the extent to which reaction goes toward the formation of the product before equilibrium is reached (Table 6). The potentiometric studies also revealed 1:2 metal-ligand ratio for the respective nickel (II) and copper (II) complexes (Table 10 and 11).

Antibacterial activity test of the schiff base and its metal (II) complexes has been determined at different concentrations. The diameter of inhibition zone $(\mathrm{mm})$ was measured for each treatment. schiff base and its complexes displayed different activities against the tested bacteria, Escherichia coli and Staphylococcus aureus (Table 7). The sensitivity of fungal isolate Aspergillus niger and Candida albican show that $\mathrm{Ni}(\mathrm{II})$ schiff base complex ismore active against Aspergillus niger, while $\mathrm{Cu}$ (II) schiff base complex is more active against Candida albicamat higher concentration.(Table 8)

From the analyses of the schiff base metal (II) complex compounds carried out the general molecular structure is proposed.

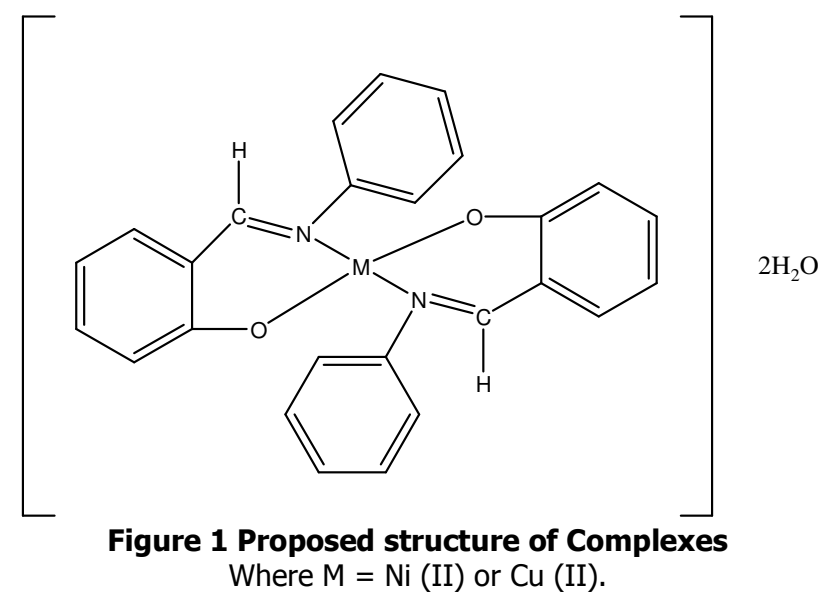

\section{CONCLUSION}

The schiff base and its complexes of $\mathrm{Ni}(\mathrm{II})$ and $\mathrm{Cu}(\mathrm{II})$ have been synthesized and characterized. The analytical data show that the metal-ligand stoichiometry in all the complexes is $1: 2$. The synthesized complexes are non-electrolyte using DMSO solvents. The spectral data show that the schiff base act as bidentate ligand coordinating through the nitrogen atoms of the azomethine and oxygen atoms of the hydroxyl group of salicylaldehyde. 
Base on analytical data the complexes are assigned to be in tetrahedral geometry. Antimicrobial studies of the schiff base ligand and complexes revealed that, the complexes are more active than schiff base.

\section{Contribution of Authors}

The work was carried out in collaboration between all the reported authors. S. Y. Hussaini designed the

\section{REFERENCES}

Avar B. Lajos T. Hoter, N and Kurt, R. (1975). "Schiff base complexes of transition metals as highest stabilizers for plastics and Fibres" Chemical Abstract 841849.

Geary WJ, Coord.chem. Rev, 1972, 1, 81.

Gregory S, Thomas BR, Robert JA (1978). Synthesis and techniques in inorganic chemistry 3rd edition, Longman publishers, London. 119126.

Hassan A. M., (1991), "Co (II) and Fe (III) Schiff base chelates derived from Isatin and some amino acids" Journal of Islamic Academy of Science, 4 (4), 271-274.

Hassan S. W, Umar R. A, Lawal M, Bilbis L S, Muhammad B. Y (2006). "Evaluation of antifungal Activity of ficus sycomores L. (Moraceae)". Best Journa/3 (2), 18-25.

Hobday M.D and Smith T.D, (1972). Coordination Chemistry Rev. 9, $311-337$.

Holm R.H (1966). "Metal Complexes of Schiff base and B. Ketamines". Inorg. Chem. 7, 83 214.

Janes D. and Kreft S (2008), "Salicylaldehyde is a characteristic aroma component of buckwheat groats". Food chemistry. 109, 293-298.

John, N.C (2002). Organic Transition metal and inorganic chemistry. Cheuron Science Centre, Pittbikg. Pg $1-2$

Makode J. T, Aswa A. S (2004) "Synthesis, characterization, biological and thermal properties of some new Schiff base complexes derived from 2-hydroxy-5chloroacetophenone and Smethyldithiocarbazate" Indian Journal of Chm. 43A. 2120-2125.

Maihab A.A., El-ajaily M.M., \& El-tajoury A.N., (2005). "Preparation and Physical Characterization of some Schiffbase Ligands derived from Salicylaldehyde and tyrosine with divalent metal ions". The Egyptian Sciences Magazine, 2 (4). 83-87. experiment and performed the laboratory work. A. Ahmadcontributed to the literature search and S. Sani partook in the results interpretation. All authors accepted the final version of the manuscript.

\section{Conflict of interest}

Authorsdeclare that, no conflict of interest.

Muhammad Basheer Ummathur, P. Sayudevi and K. Krishnan kutty(2008)."Metal complexes of Schiff bases derived from dicinnamoylmethane and aromatic amines".Journal of the Agentine chemical society, 96, 13-21.

Morad F. M, A.N. El - Tajoury and M.M. El-ajaily, (2006). "Chelation behaviour and biological,the activity of divalent metal ions towards Schiff base". Basic Science and its application J. 1 (1) $196-210$.

Nazeeruddin M.K., Gratzel M. (2007) "Transition Metal Complexes for Photovoltaic and Light Emitting Applications". Photofunctional Transition Metal Complexes. Structure and Bonding, 123. 113-175. Springer, Berlin, Heidelberg

Neelakandan, M. A.,Raman N. And Dhaveethuraja J, (2008), "DNA Cleavage and Antimicrobial Activity Studies on Transition Metal (II) Complexes". Journal of the Chilean chemical society. 41, 399-410.

Saleem, H. S., El-Shetary, B. A. and Khalil S. M. (2003) "Potentiometric and Spectrophotometric Studies of Complexation of Schiff base Hydrazine containing the pyrimidine moity" J. Serb. Chem. Soc., 68(10) $729-748$.

Shamsudden U, Mukhtar MD, Salisu M (2008). "Comparative in-vitro study of Activity of Methanolic and Ethanolic extracts of onion seeds on gram-negative and gram-positive bacteria". Best journals. 5(3)17-20.

Sharma R.C and Mohan G, (1990), the biological activity of some Schiff bases and its complexes, Chemical Abstract. 113, 165240.

Silver S. S.M and Bassler G.C (1967) Spectrophotometric identification of organic compounds, Wiley, New York 12-23.

Vogel A.I (1972). Quantitative inorganic analysis including elementary instrumental analysis $3^{\text {rd }}$ Edition Longman co. London Pg. 526-535. 